MS3-P8 Getting the Most of the Sample with Small Beams and Fast Detectors

Martin Savko ${ }^{1}$, Gavin Fox ${ }^{1}$, Enrico Stura ${ }^{1,2}$, William Shepard ${ }^{1}$

1. Synchrotron SOLEIL, France

2. Departement d'Ingenierie et d'Etude des Proteines, CEA, Saclay, France

email: savko@synchrotron-soleil.fr

Combining fast sensitive detectors, high performance goniometers and small X-ray beams opens new venues in exploring the information content in the samples.

The photon budget available to explore the sample is limited, and to fully benefit from a fortuitous combination, the experimenter needs to weigh up the characteristics of the beam, the detector and the sample.

Holistic approaches to sample characterization, which combine optical imaging with X-ray techniques (fluorescence, diffraction and tomography), has a potential to provide a fuller appreciation of the spatial distribution of the sample properties and can provide a useful guidelines in the design of the experiment.

The increased data flow from the fast detectors also poses the new challenges for real time evaluation, and the computing infrastructure, which has always been important in the macromolecular crystallography experiments, becomes even more essential component in the overall workflow.

Here we present the practical choices made and the current solutions available on the Proxima 2A microfocus beamline at Synchrotron SOLEIL for the experiments performed using the newly commissioned Eiger X $9 \mathrm{M}$ detector.

Keywords: microbeam, Eiger, data processing

\section{MS3-P9 The automated expert processing system XDSAPP}

Karine M. Sparta ${ }^{1}$, Uwe Mueller ${ }^{2}$, Udo Heinemann ${ }^{3,4}$, Manfred S. Weiss ${ }^{1}$

1. Helmholtz-Zentrum Berlin, Macromolecular Crystallography, Berlin, Germany

2. MAX IV Laboratory, Lund University, Lund, Sweden

3. Macromolecular Structure and Interaction, Max Delbrück Center for Molecular Medicine, Berlin, Germany

4. Chemistry and Biochemistry Institute, Freie Universität Berlin, Berlin, Germany

email: karine.sparta@helmholtz-berlin.de

In the last decade, macromolecular crystallography (MX) has experienced constant improvements of beamline efficiency at synchrotron sources around the world, leading to exposure times below one second per image and complete data collection within a few minutes. At the Helmholtz-Zentrum Berlin (HZB), we operate three MX beamlines, two of which are equipped with Pilatus detectors and sample changers [1], enabling fast high throughput experiments to be performed routinely.

We have developed the expert software XDSAPP (XDS Automation and Plotting Protocols) [2] to support users in the analysis of their diffraction data during measurement at our beamlines with minimal effort and time. It constitutes a convenient interface to XDS [3] and further relevant software such as POINTLESS from the CCP4 suite [4, 5], XDSSTAT [6], SFCHECK [7] and PHENIX.XTRIAGE [8], needed for automated decision-making. All important statistics from the XDS output files are represented graphically.

Originally developed as a $\mathrm{Tcl} / \mathrm{Tk}$ graphical user interface (GUI), a completely new version has been made available for download since February 2014. Its new GUI has been designed to provide users with a much simplified and more intuitive way of handling diffraction data sets [9].

XDSAPP is used in more than 500 research groups distributed in 43 countries and is freely available to academic users from www.helmholtz-berlin.de/bessy-mx.

References

[1] Mueller, U. et al. (2015). Eur. Phys. J. Plus 130, 141-150.

[2] Krug, M. et al. (2012). J. Appl. Cryst. 45, 568-572.

[3] Kabsch, W. (2012). Acta Cryst. D66, 125-132.

[4] Collaborative Computational Project (1994). Acta Cryst. D50, 760-763.

[5] Winns, M.D. et al. (2011). Acta Cryst. D67, 235-242.

[6] Diederichs, K. (2006). Acta Cryst. D62, 96-101.

[7] Vaguine, A. et al. (1999). Acta Cryst. D55, 191-205.

[8] Adams, P.D. et al. (2010). Acta Cryst. D66, 213-221.

[9] Sparta, K. et al. (2016). J. Appl. Cryst. 49.

Keywords: XDSAPP, software, data processing 